\title{
Ca II HK emission in rapidly rotating stars
}

\section{Evidence for an onset of the solar-type dynamo}

\author{
C. Schröder ${ }^{1}$, A. Reiners ${ }^{2}$, and J. H. M. M. Schmitt ${ }^{1}$ \\ 1 Hamburger Sternwarte, Gojenbergsweg 112, 21029 Hamburg, Germany \\ e-mail: cschroeder@hs .uni-hamburg.de \\ 2 Georg-August-Universitaet, Friedrich-Hund-Platz 1, 37077 Göttingen, Germany
}

Received 12 June 2008 / Accepted 11 August 2008

\section{ABSTRACT}

\begin{abstract}
We present measurements of chromospheric Ca II H\&K activity for 481 solar-like stars. To determine the activity we used the Mount Wilson method and a newly developed method which allows to also measure Ca II H\&K emission features in very rapidly rotating stars. The new technique determines the activity by comparing the line shapes from known inactive slowly rotating template stars that have been artificially broadened to spectra of rapid rotators. We have analyzed solar-like stars ranging from $T_{\text {eff }}=5000$ to $7800 \mathrm{~K}$ with rotational velocities up to $190 \mathrm{~km} \mathrm{~s}^{-1}$ in our sample of FOCES and FEROS spectra. The effects of the rotational broadening on the two methods have been quantified. Our method has proven to produce consistent results where $S$-Index values are available and offers the possibility to measure the chromospheric activity at the onset of the solar-like dynamo.
\end{abstract}

Key words. stars: activity - stars: chromospheres - stars: rotation

\section{Introduction}

Since Olin C. Wilson began his research of stellar activity in 1966 (Wilson 1978), many activity-related projects have been carried out using the Mt. Wilson $S$-index as a measure for chromospheric activity (Vaughan et al. 1978; Duncan et al. 1991; Baliunas et al. 1998). From 1966 to 1977 the Mount Wilson HK program was carried out with the so-called HKP-1 photometer, which contained a photoelectric scanner at the Coudé focus of the 100 inch telescope. From 1977, the survey was continued with a specially designed photomultiplier, the HKP-2, mounted on the Cassegrain focus of the 60 inch telescope (Baliunas et al. 1995). The data collected during the "HK Project" laid the groundwork for our current understanding of stellar chromospheric activity.

Further surveys contributed to the pool of activity measurements. Henry et al. (1996) surveyed over 800 southern Sun-like stars and identified four activity classes in their sample, ranging from very inactive to very active. Between the inactive and active regime they found a deficiency of stars at intermediate levels of activity, which was confirmed by Gray et al. (2003, 2006) in their NStars project. Another large survey was presented by Wright et al. (2004), who studied the $S$-index, $R_{\mathrm{HK}}^{\prime}$, chromospheric ages and rotation rates for over $1200 \mathrm{~F}$ to $\mathrm{M}$ stars. Since 1994, long-term observations obtained with Solar-Stellar Spectrograph (SSS) at Lowell Observatory measured the $S$ index and absolute flux of the Sun and solar-like stars (Hall et al. 2007).

All of these surveys are based on the same method as used for the Mount Wilson observations. This technique measures the chromospheric activity by determining the flux in $\approx 1 \AA$ wide

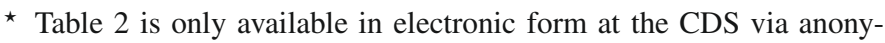
mous ftp to cdsarc.u-strasbg. fr $(130.79 .128 .5)$ or via http://cdsweb.u-strasbg.fr/cgi-bin/qcat?J/A+A/493/1099 filters in the centers of the Ca II H\&K lines. The measured flux is then normalized to the flux in two continuum filters placed shortward and longward of the Ca II H\&K lines. A known drawback of this method is the fact that it can only be applied to slowly rotating stars. For the rapid rotators the wings of the $\mathrm{Ca}$ II $\mathrm{H} \& \mathrm{~K}$ lines wings fill up the center of the absorption line and mimic emission flux, and also emission line flux in the core is lost due to rotational line broadening. Since, first, according to the rotation-activity paradigm the fast rotators should be the stars with the largest activity, and second, fast rotators are often found among the late A and F-type stars, it is highly desirable to have a method producing an $S$-index value also for such objects. In addition, with the currently available high resolution échelle spectrographs, which often cover the whole visual wavelength range, many serendipitous observations of the $\mathrm{Ca}$ II $\mathrm{H} \& \mathrm{~K}$ lines are frequently available, which can be utilized for surveys of chromospheric activity. In this paper we therefore present a new method to measure $\mathrm{Ca}$ II $\mathrm{HK}$ emission features in very rapidly rotating stars by comparing the line shapes from known inactive slowly rotating template stars that have been artificially broadened to those of fast rotators.

In Sect. 2, we present the observational data used to measure the Ca II activity and the methods applied to obtain the basic data for our stars. Section 3 contains the descriptions of the classical Mount Wilson method and our new template method. In Sect. 4 we analyze the influence of rotational broadening on the classical and the new template $R_{\mathrm{HK}}^{\prime}$ values and present the results of our activity measurements. Section 5 contains a summary and the conclusions.

\section{Observational data}

The sample of spectra we used for our Ca II H\&K measurements is comprised of three different subsamples of archived data originally acquired for different scientific purposes. The 
first collection of 74 spectra was observed in April 2002 with the FEROS instrument at the ESO $1.52 \mathrm{~m}$ telescope at La Silla. FEROS is an échelle spectrograph (Kaufer et al. 1999) with a resolution of $R=48000$ providing an almost complete spectrum in the range of 3500 to $9200 \AA$. The second sample of 155 spectra was observed in December 2003 and April 2004, again with the FEROS instrument, which in the meantime had been moved to the MPG/ESO $2.2 \mathrm{~m}$ telescope to enhance performance. This collection of spectra was part of a radial velocity measurement program from Setiawan. Due to this scientific goal, this sample of stars is biased towards slower rotational velocities. Both FEROS spectra samples yield a signal-to-noise ratio of at least 200 in the continuum around $6000 \AA$. The last collection of 252 spectra was observed in February 2002, March 2004 and August 2004 with FOCES at the $2.2 \mathrm{~m}$ telescope on Calar Alto (Spain). FOCES is again an échelle spectrograph, that can be mounted at the Cassegrain focus on either the $2.2 \mathrm{~m}$ or $3.5 \mathrm{~m}$ telescope at the DSAZ (Calar Alto, Spain). Its spectral resolution reaches, depending on the setup, up to $R=40000$. The signalto-noise ratio in this sample is at least 100 in the continuum. We note a possible bias towards higher rotational velocities in the first FEROS and the FOCES sample, which have been observed in a campaign to study differential rotation. In this campaign stars with a rotational velocity of $30 \mathrm{~km} \mathrm{~s}^{-1}$ and more have been observed with high priority, while the slow rotators were added later during the campaign. Therefore, we cannot rule out a bias towards higher rotational velocities in two of our three subsamples. A detailed description of the observations can be found in Reiners \& Schmitt (2003a).

Since our method uses spectra of inactive template stars to measure the Ca II H\&K activity and the templates are selected based on $T_{\text {eff }}$ and $\log g$ of the star, these fundamental parameters are required for all stars in our sample. We used literature data whenever available, and to determine the required values for those stars without literature data, we used tools based on uvby $\beta$ photometry. Moon \& Dworetsky (1985) compared the observed colours and indices for B, A and F stars of known $T_{\text {eff }}$ and $\log g$ with synthetic colors, calculated by Relyea \& Kurucz (1978) and Schmidt \& Taylor (1979). Further improvements on the grids used in this method have been applied by Napiwotzki et al. (1992). The determined effective temperatures in our sample range from 5000 to $7800 \mathrm{~K}$ and are in good agreement with the values given by Nordström et al. (2004).

The $v \sin (i)$ values of our sample have been determined using a Least Square Deconvolution method (LSD), which is based in the analysis of the zeros of the Fourier transformed line profile; further details about the extraction of $v \sin (i)$ from high resolution spectra can be found in Reiners \& Schmitt (2002, 2003b). For the slowest rotators $\left(v \sin (i) \leq 4 \mathrm{~km} \mathrm{~s}^{-1}\right)$, the application of this method becomes difficult and the measurements represent upper limits. The $v \sin (i)$ values not listed by Reiners \& Schmitt (2002, 2003b), were taken from the Glebocki \& Stawikowski (2000). In Fig. 1 we shows the $v \sin (i)$ values vs. the $B-V$ color index in our sample; as is expected and obvious from Fig. 1, there exist many rapid rotators among the stars with $B-V<0.6$, for which one would like to obtain a quantitative measurement of their chromospheric activity.

The classical Mount Wilson method to measure the $\log R_{\mathrm{HK}}^{\prime}$ values cannot be applied to evolved stars, since the photospheric contribution to the measured flux has not been determined for giants. In these objects the photospheric contribution is lower than in main-sequence stars. Since the depths of the absorption and therefore the photospheric correction depends on the

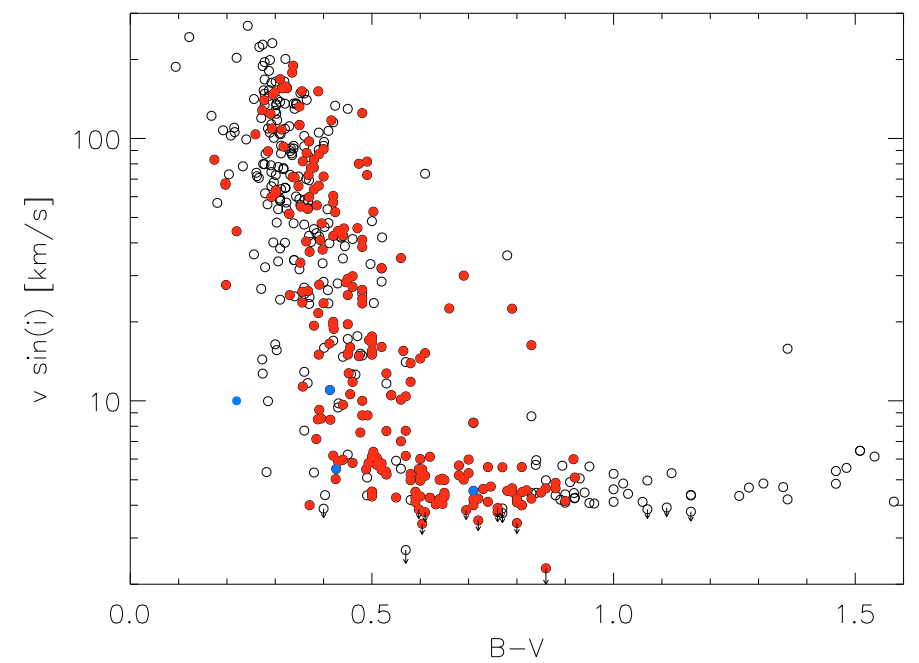

Fig. 1. Rotational velocity plotted over $B-V$ color for the 481 stars in our data sample. The red colored symbols represent those 238 stars with activity measurements based on high-quality fits of the star spectrum on an inactive template star spectrum. Indicated by the filled blue circles are stars which have been chosen as template stars.

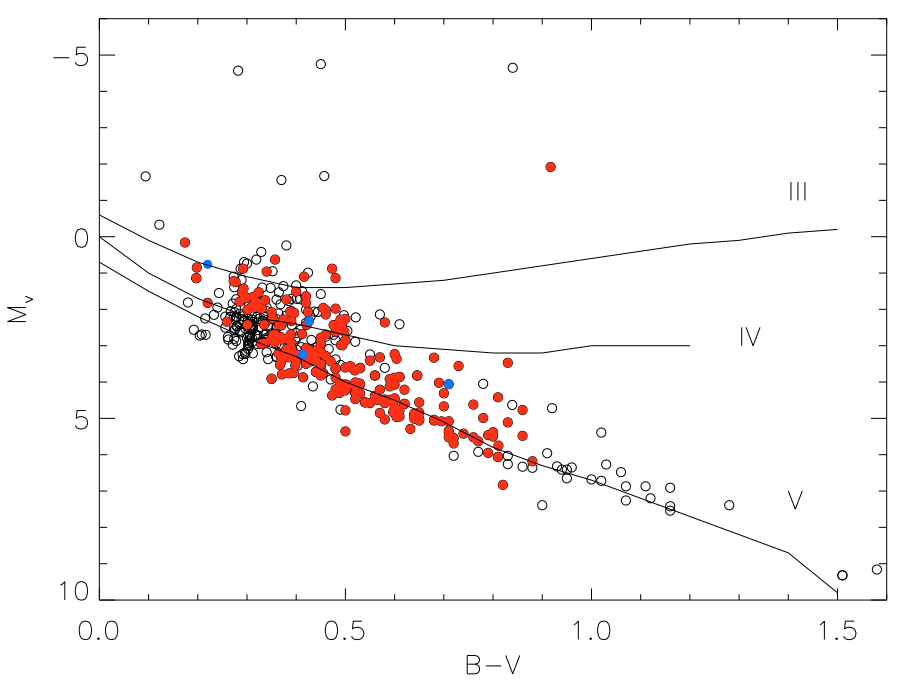

Fig. 2. Color-magnitude diagram for the 481 stars in our data sample. The black circles represent the 481 stars with activity measurements with the Mount Wilson method. The filled red circles are those stars with an additional template method measurement and stars chosen as templates are represented with filled blue circles. Indicated by the solid lines are the luminosity classes according to Allen (1976).

surface gravity, the photospheric correction for giants has to be determined for different evolutionary stages, which has not been done so far. To identify the evolved stars in our sample, we determined their position in the Hertzsprung-Russell diagram (see Fig. 2). The absolute luminosities were taken from Nordström et al. (2004) or calculated based on the Hipparcos parallaxes for the stars not listed in Nordström et al. (2004). We plotted the luminosity classes according to Allen (1976) as solid lines. All stars brighter than luminosity class IV are treated as giant stars with respect to the classical method. For these stars, we only give the $S$ values of the classical method and not the $\log R_{\mathrm{HK}}^{\prime}$. Stars with luminosity class V to IV are treated as main-sequence stars in terms of the application of the Mount Wilson method and are listed with both the $S$ index and the $\log R_{\mathrm{HK}}^{\prime}$ values. 


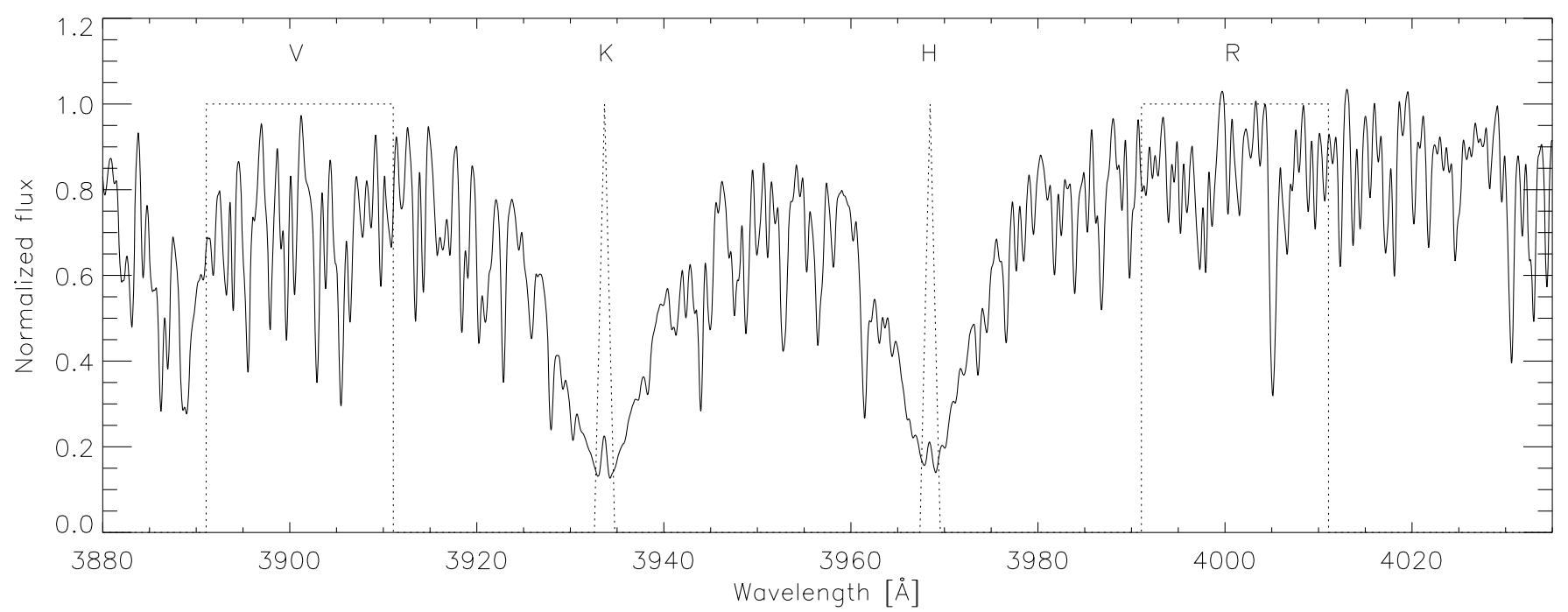

Fig. 3. The two triangle-shaped line filters and the continuum filters used to calculate the Mount Wilson S index shown in a normalized FEROS spectrum of the slowly rotating F7V stars HD 48676.

In an unresolved binary system the presence of a companion might increase the apparent luminosity of the primary to a value which would lead to a classification of the primary as an evolved star. As shown in Fig. 4, the difference between the conversion factors given in Eqs. (3) and 4 is small for stars with $B-V<1.0$. Since the evolved stars in our sample are located at $B-V<1.0$, the effect of such companions has no significant influence on our measurements.

\section{Method description}

\subsection{Classical S-index, $\log R_{H K}^{\prime}$ and the Rossby number}

The $S$-index was introduced by Vaughan et al. (1978) as a dimensionless indicator for the Ca II activity measured by the Mount Wilson Observatory (MWO) HKP-1 and HKP-2 spectrometers. The HKP-2 instrument, which laid the groundwork for following Ca II surveys, measured the flux in two $1.09 \AA$ wide $H$ and $K$ bandpasses and normalized it to the flux in two $20 \AA$ wide continuum filters placed at 3901 and $4001 \AA$, referred to as $V$ and $R$; in Fig. 3 we show the position and the size of the various filters for the case of the slow rotator HD 48676. The $S$-index values were constructed as

$S=\alpha \frac{H+K}{R+V}$,

where $H, K, R$, and $V$ are the values for the flux measured in the according bandpasses (cf., Fig. 3) and $\alpha$ is an instrumental calibration factor. This calibration is necessary to adjust the measured activity indices from the two spectrometers HKP-1 and HKP-2. Values for $\alpha$ range from 1.3 (Cincunegui et al. 2007) to 5 (Gray et al. 2003).

Since the flux measured in the core of the Ca II lines is normalized to the continuum flux, the $S$-index is independent from the color of the star. Clearly, in the hypothetical case of a totally inactive star there is also a photospheric contribution to the $\mathrm{H}$ and $\mathrm{K}$ flux measurements and hence the $S$-index. To remove the color independence and the photospheric component, Middelkoop (1982) developed a transformation of the $\mathrm{S}$ index into a value $R_{\mathrm{HK}}$ as a function of $B-V$ based on 85 main sequence stars:

$R_{\mathrm{HK}}=1.34 \times 10^{-4} C_{\mathrm{cf}} S$.
Rutten (1984) improved this relation for the conversion factor $C_{\mathrm{cf}}$ given by Middelkoop (1982) and extended the range of observed stars to $B-V=0.3$. With the additionally observed 30 main-sequence and 27 giant stars, they found improved fits through

$\log C_{\mathrm{cf}}=0.25(B-V)^{3}-1.33(B-V)^{2}+0.43(B-V)+0.24$

for main-sequence stars with $0.3 \leq B-V \leq 1.6$, and

$\log C_{\mathrm{cf}}=-0.066(B-V)^{3}-0.25(B-V)^{2}-0.49(B-V)+0.45(4)$

for giants with $0.3 \leq B-V \leq 1.7$. The improvements in this new relation is based on the larger number of stars, especially on the blue side of the samples, and the underlying relations for $B-V$, $T_{\text {eff }}$ and $B C$. A comparison between the conversion factors given by Rutten (1984) and Middelkoop (1982) is shown in Fig. 4. As the plot shows, the difference between the conversion factors is small, in particular for hotter stars. We used the relation given by Rutten (1984), since it was determined based on a broader color range.

To correct for the photospheric contribution to the flux in the Ca II line cores Noyes (1984) derived the expression

$\log R_{\text {phot }}=-4.898+1.918(B-V)^{2}-2.893(B-V)^{3}$

in the range of $0.44\langle(B-V)\rangle 0.82$, which provides the photospheric term for the correction

$R_{\mathrm{HK}}^{\prime}=R_{\mathrm{HK}}-R_{\mathrm{phot}}$.

For $B-V>0.82$ the photospheric contribution is still in good agreement with Eq. (5) and, as reported by Noyes (1984), becomes negligible for $B-V \geq 1$. Noyes (1984) found an empirical fit between the Rossby number and the emission ratio $R_{\mathrm{HK}}^{\prime}$ through

$$
\begin{aligned}
\log \left(P / \tau_{c}\right) \equiv f\left(R_{\mathrm{HK}}^{\prime}\right)= & 0.324-0.4 \log R_{5}-0.283\left(\log R_{5}\right)^{2} \\
- & 1.325\left(\log R_{5}\right)^{3}
\end{aligned}
$$

where $R_{5}$ is defined as $R_{\mathrm{HK}}^{\prime} \times 10^{5}$ and $\tau_{c}$ is the convective turnover time based on the ratio of mixing length to scale height $\alpha=2$. By placing a fit to a $\log P_{\mathrm{obs}}-f\left(R_{\mathrm{HK}}^{\prime}\right)$ over $B-V$ plot, they derived a corrected convective turn-over time

$$
\log \tau_{c}^{(2)}=\begin{array}{ll}
1.362-0.166 x+0.025 x^{2}-5.323 x^{3}, & x>0 \\
1.362-0.14 x, & x<0 .
\end{array}
$$




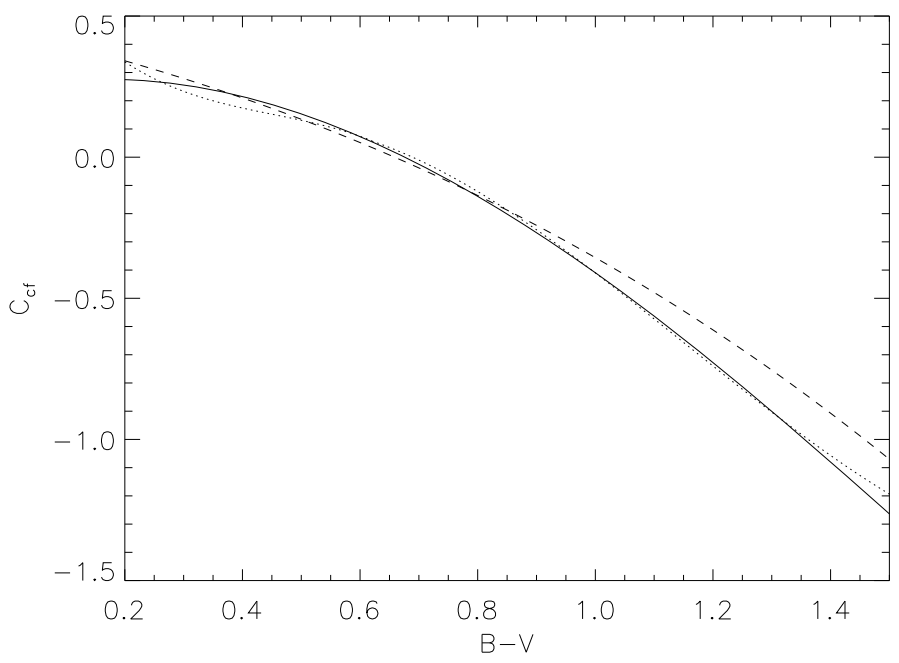

Fig. 4. Comparison of the different conversion factors as a function of $B-V$. The solid line shows the conversion factor for main-sequence stars (specified in the range of $0.3 \geq B-V \leq 1.6$ ), the dashed line

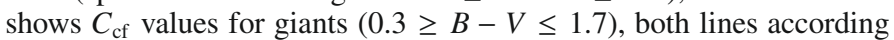
Rutten (1984), and the dotted line the values according to Noyes (1984) for the main-sequence $(0.45 \geq B-V \leq 1.5)$.

In this case, $x$ is defined as $x=1-(B-V)$ and the ratio of mixing length to scale height is 1.9. Noyes (1984) found that the correlation between $R o$ and $\log R_{\mathrm{HK}}^{\prime}$ shows the least dispersion for this particular value of $\alpha$. With the convective turnover time $\tau_{c}^{(2)}$ and and a given rotational period $P$ it is possible to define a dimensionless Rossby number $R o=P_{\text {obs }} / \tau_{c}^{(2)}$, which was determined empirically by Noyes (1984) and allows a significantly better description of the chromospheric activity than rotational velocity or rotation period alone.

\subsection{Template method}

A known problem of the Mount Wilson $S$-index is the fact that for rapidly rotating stars the measured $S$-index is influenced by the broadening of the line wings and the core emission feature. This rotational effect limits the classical method to slowly rotating stars. Especially activity measurements of rapidly rotating late A- and early F-type stars, where the onset of the magnetic dynamo occurs, are susceptible to rotational broadening. Figure 1 clearly shows the well-known increase of the rotational velocities for the stars with $B-V<0.5$, so clearly one needs a way to obtain $S$-indices independent of rotation of a star.

The idea behind our method is the fact that even in highly broadened spectra the difference between active and inactive stars should be detectable, given a sufficiently high signal-tonoise ratio in both spectra and a reasonable agreement in $T_{\text {eff }}$ and $\log g$. The calibration of the spectra and the measurement of the activity are divided into several steps. First, both the target and template stellar spectra are normalized and wavelength calibrated in the usual way. In a second step, the template spectrum is normalized with a first order polynomial to the stars spectrum in the wings of the $\mathrm{H}$ line. Here we focused on the $\mathrm{Ca}$ II H line, because the $\mathrm{K}$ line is much more sensitive to differences between the template and target star in $\log g$ and $T_{\text {eff. }}$. We obtain this polynomial by calculating the ratio between the template and the star spectrum in a number of $0.5 \AA$ wide areas. These areas are equally spread over two $4.5 \AA$ wide regions, which are located on the red and blue side of the line and cover the

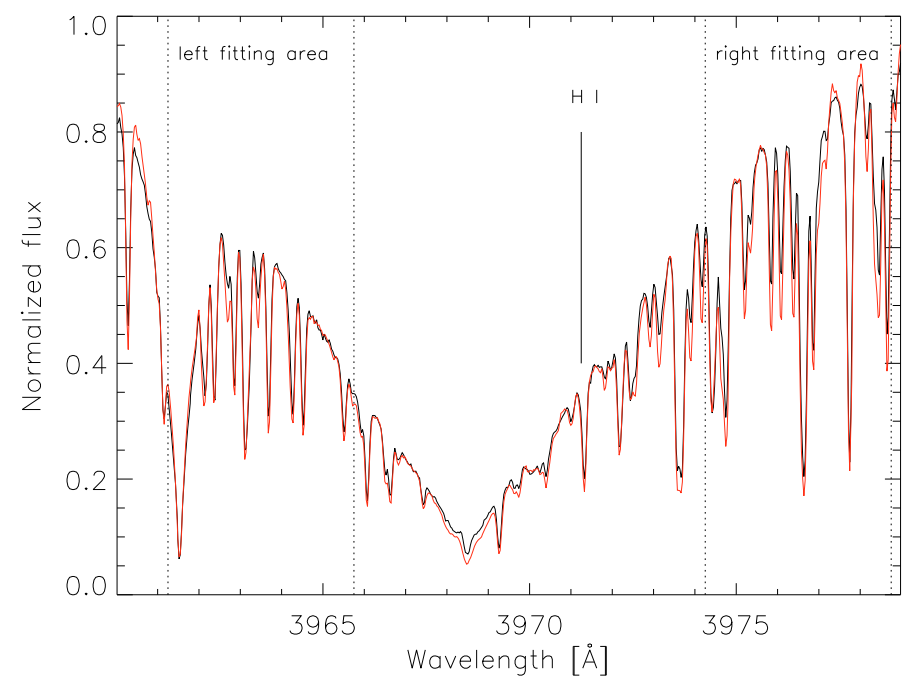

Fig. 5. An example of a fitting result for the slowly rotating G2V template star HD 128620 to the G2V star HD 102365. Shown in the plot are the areas for the foot points for the line wing fitting and the position of the $\mathrm{H}$ I line at $3971.2 \AA$. The difference in $\log g$ is 0.7 and there is no significant temperature difference.

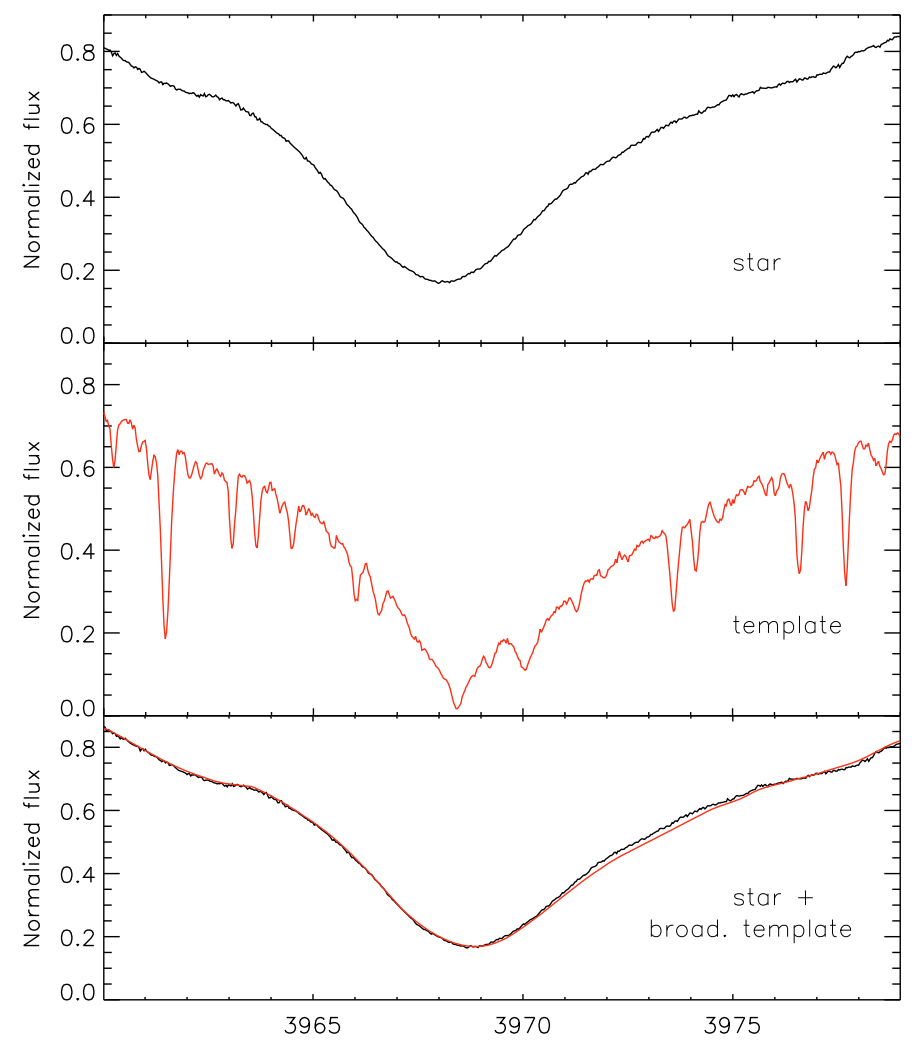

Fig. 6. An example of a fitting result for a rapidly rotating star. From top to bottom: the F3IV-V star HD 186005, the F0IV template star HD 36876 and the result of the fitting process. The differences are $580 \mathrm{~K}$ in $T_{\text {eff }}$ and 0.14 in $\log g$, and the rotational velocity of HD 186005 is $150 \mathrm{~km} \mathrm{~s}^{-1}$.

wavelength range from 3961.25 to $3965.75 \AA$ in the blue wing and from 3974.25 to $3978.75 \AA$ in the red wing. Both fitting areas are indicated in Figs. 5 and 6 . The right fitting region is positioned on the far wing of the $\mathrm{K}$ line to prevent any influence of the H I line at $3971.2 \AA$ on the measurements of the hottest stars in our sample. 
In the third step we identify the maxima for both the template and the star spectrum in each of the eighteen $0.5 \AA$ wide fitting areas and calculate their ratio. Since the mean or the median are influenced by differences of the line depths caused by different metallicities of the template and the star, the maxima lead to better fit results. The polynomial is then obtained as a first order fit to this array of ratios. By dividing the template by this polynomial in the appropriate wavelength range, we correct for the last differences between the two spectra remaining after the normalization of the template and the target star's spectrum. The results of the fitting process is shown in Figs. 5 and 6. In Fig. 5 the slowly rotating G2V star HD 102365 is compared to the template star HD 128620. Since both stars are slow rotators, the fitting process does not include any artificial broadening. For the F3IVV star HD 186005, which rotates with $150 \mathrm{~km} \mathrm{~s}^{-1}$, the template spectrum has to be strongly broadened. Figure 6 shows the spectra of the target star and the template before the fitting process in the top and center plot, as well as the result of the broadening and fitting process in the bottom plot.

To measure the Ca II H\&K emission in the fourth step, the integral over a given area in center of the line was calculated for the target star and the template. The extraction area has to be large enough to cover even strongly broadened emission features. To reduce the effect of different metallicities, we chose a variable extraction width of at minimum $2 \AA$ width around the center of the line for very slowly rotating stars, and broaden the extraction width according to the red- and blueshift of the rotational velocity. Since the wings of the $\mathrm{Ca}$ II $\mathrm{H}$ line is populated with various Fe I lines, the variable extraction width reduces the noise in the measured emission. To exclude any possibly remaining $B-V$ dependent effects and stay consistent with the classical $S$ values, the difference of the two integrals is then normalized to the equivalent width of the continuum in two continuum filters (see Fig. 3). These continuum filters are the same as in the $S$-index calculations presented by Vaughan et al. (1978). To stay as close as possible to the classical $S$ index, we then multiplied our re-

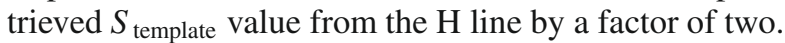

To convert our $S$-indices into the color dependend $\log R_{\mathrm{HK}}^{\prime}$ values in the last step, we used the conversion factor $C_{\mathrm{cf}}$ from Rutten (1984) given in Eq. (3), since a significant fraction of our sample is located in the range of $B-V \leq 0.4$. For these stars, the conversion factor presented by Rutten (1984) is based on more observational data and therefore better defined values for the hotter stars than the conversion factor presented by Middelkoop (1982) and Noyes (1984). For the giants in our sample we used Eq. (4). Since our method already substracts the photospheric contribution to the flux with the template, we do not need to correct for the photospheric flux.

The template stars and their $T_{\text {eff }}, \log g$ and $v \sin (i)$ values are given in Table 1. The selection criteria for these stars were slow rotational velocity and that the stars should be as inactive as possible. Additionally, the $\log g$ values of the templates cover a large fraction of the $\log g$ values given in our sample. The application of these criteria resulted in the selection of the given four template stars. With this selection of template stars we are able to measure $S$-indices in about $50 \%$ of the stars in our sample. For the stars without template measurements the difference in $\log g$ and $T_{\text {eff }}$ is too large to give an acceptable fit result. Additional templates covering the missing values of $\log g$ and $T_{\text {eff }}$ would allow us to measure a large fraction of the missing stars. A possible source for template spectra are obviously synthetic spectra. We actually did compute the missing spectra with PHOENIX (Hauschildt \& Baron 1999), however, we found systematic differences in the line wings between synthetic and observed
Table 1. List of template stars.

\begin{tabular}{lcclcc}
\hline \hline HD & $T_{\text {eff }}$ & $\log g$ & $v \sin (i)$ & Temperature range & Id \\
\hline 128620 & 5627 & 4.17 & 4.5 & $<6200 \mathrm{~K}$ & 1 \\
143790 & 6270 & 3.93 & 5.5 & $6200-6900 \mathrm{~K}, \log g<4$ & 2 \\
104731 & 6572 & 4.26 & 11.0 & $6200-6900 \mathrm{~K}, \log g>4$ & 3 \\
36876 & 7567 & 3.67 & 10.0 & $>6900 \mathrm{~K}$ & 4 \\
\hline
\end{tabular}

spectra and therefore chose to only use observed template spectra.

\subsubsection{Uncertainties}

Based on visual inspection of the individual fit results we estimate an uncertainty of about 0.03 for our derived $S_{\text {template }}$ values. This uncertainty is caused by noise in the spectra, especially in the line core, inaccuracies in the spectral fits and differences in $\log g$ and $T_{\text {eff }}$ between the template and the target star. In the following we analyze the different contributions to the measurement errors.

We investigate the impact of the noise on the measured activity by adding a artificial noise to a high quality fit. The effect of the noise is that the template is fitted to the noise peaks in the line wings (see Sect. 3.2), which leads to a lower activity measured in the line core. If we lower the signal-to-noise ratio to a value of 10 for HD 102365, we find a decrease of the $S_{\text {template }}$ value by 0.003 compared to the original spectrum, which leads to the conclusion that the noise is only a minor fraction of error.

To test the influence of the difference in $\log g$ and $T_{\text {eff }}$ and therefore the effect of an inadequate template, we measured the activity for deliberately poorly fitting templates. For the stars presented in Figs. 5 and 6, HD 102365 and HD 186005, we found variations of the measured $S_{\text {template }}$ of 0.01 if we choose the next hotter or cooler template respectively. Going one step further and using the hottest template star HD 36876 with $T_{\text {eff }}=7567 \mathrm{~K}$ to measure HD $102365\left(T_{\text {eff }}=5624 \mathrm{~K}\right)$ results in $S_{\text {template }}=0.036$. Changing the template to a lower $\log g$ results in a variation of $S_{\text {template }}$ of 0.01 for the highest difference of 0.95 . Hence, the selection of the template and therefore the difference in temperature and surface gravity, is the largest contribution to the uncertainty.

According to the given contributions to the uncertainty, we make the conservative assessment that stars with an emission excess $S_{\text {template }} \leq 0.03$ still count as inactive as the selected template star. The application of Eqs. (3) and (4) results in $\log R_{\mathrm{HK}}^{\prime}$ (template) values from -5.5 to -5.0 for our most inactive stars, depending on the color of the target star. For the Sun Baliunas et al. (1995) derive a $S$-index of 0.179 , which results in a $\log R_{\mathrm{HK}}^{\prime}$ (template) value of -4.9 and is thus in line with the derived $S$-index levels of our low activity sample stars.

\section{Results}

In the following section, we present the results of the measurements with the Mount Wilson method, which were obtained to compare the classical method with the results of our new template method. The results of the MWO method a presented in Sect. 4.1. Additionally, the influence of the rotational broadening on the $\log R_{\mathrm{HK}}^{\prime}$ values and a comparison between the Mount Wilson $S$-index and the template $S$-index determinations are discussed in Sect. 4.2. 


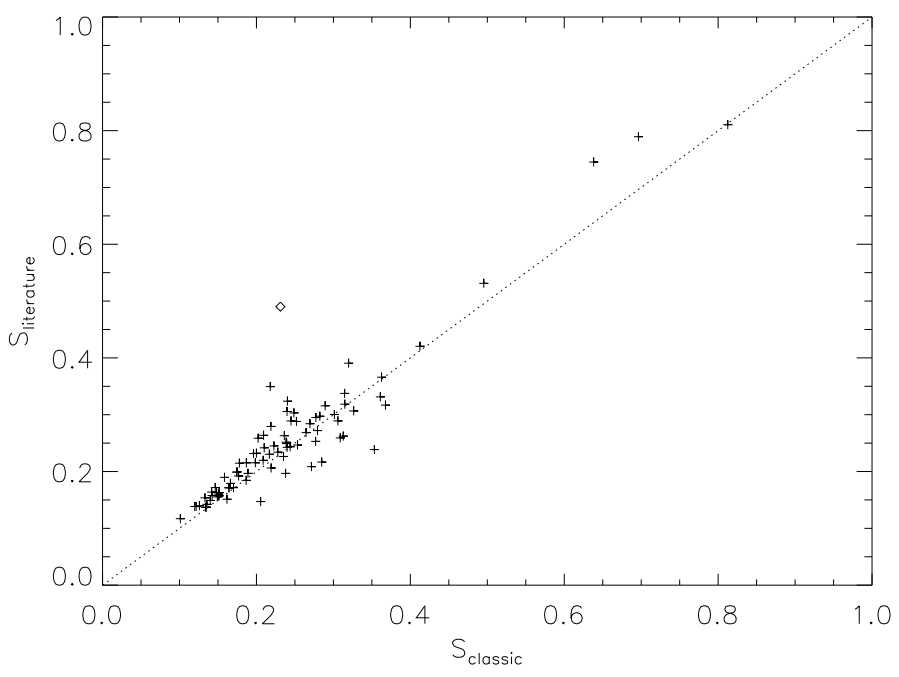

Fig. 7. $S$ values for our sample determined with the Mt Wilson method plotted over the $S$ values presented in the literature. The diamond symbol represents the BY Dra-type variable $\epsilon$ Eri.

\subsection{Comparison to previous measurements}

To determine the reliability of our new activity measurements we calculated the $S$-indices via the Mt. Wilson method for all of our sample stars. The $H$ and $K$ filters were simulated by an appropriate triangular shaped function. After multiplying the spectrum with the filter function, we normalize the integral of the $\mathrm{Ca}$ II $\mathrm{H}$ and $\mathrm{K}$ lines with the integral over the continuum filters $V$ and $R$. To determine the factor $\alpha$ in Eq. (1) for our data, we performed a linear least-squares fit between the $S$-indices of stars with previous measurements presented in Baliunas et al. (1995) and our newly calculated (classical) $S$-indices. For our sample stars we require an $\alpha$-value of 1.07 to obtain $S$-indices consistent with the published literature data. The result of our calibration is presented in Fig. 7. As the plot shows, the classical measurements are in good agreement with the literature data. We attribute the remaining rms scatter of $14 \%$ to the intrinsic variability of the stars.

This interpretation is supported by a detailed analysis of the star with the largest discrepancy. Represented by the diamond symbol in Fig. 7 is the BY Dra-type variable $\epsilon$ Eri, which is known to be a highly variable object. From our data we obtained an $S$-index of 0.23 , which is significantly lower than previously published values. Duncan et al. (1991) presented data from more than 1000 observations, in which the $S$-indices varied from 0.49 to 0.64 , with the higher values towards the beginning of the observations around 1970 and the lower values concentrated around 1980. However, Henry et al. (1996) found $S$-indices of $\sim 0.35$ in the spectra observed in 1992 and 1993 with the Cerro Tololo InterAmerican Observatory (CTIO), and Hall et al. (2007) presented observations from 1994 to 2006, which are in agreement with the data from Henry et al. (1996). With our measurement of $S=0.23$ we seem to have measured $\epsilon$ Eri in an exceptionally low state of activity. Note in this context that the presented $S$-index is based on a single measurement, while the literature data represent on a large number of observations.

\subsection{Influence of rotational broadening}

The main advantage of our new method is the fact that it returns precise $S$-indices also for rapidly rotating stars. This is demonstrated in Fig. 8, where we compare the $\log R_{\mathrm{HK}}^{\prime}$ values

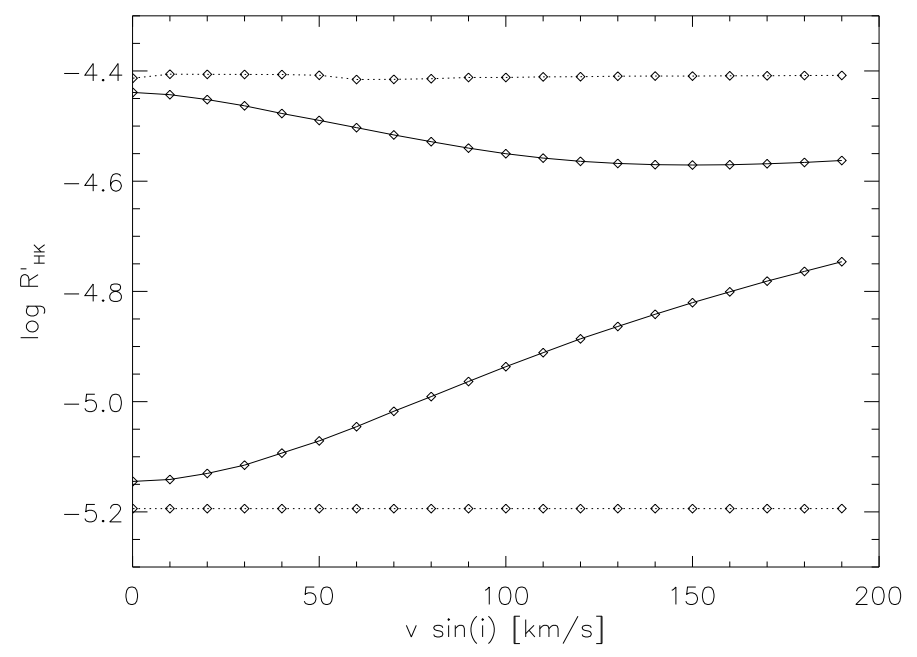

Fig. 8. Projected rotational velocity vs. chromospheric activity in $\log R_{\mathrm{HK}}^{\prime}$ measured with the classical Mt. Wilson method and the template method. The solid lines represent the data for the classical method and the dotted lines show the values for the template measurements. As the inactive star, we chose the template star HD 128620. To show the influence on an active star, we included an artificial Ca II H\&K emission feature in the spectrum of HD 128620.

obtained from two different methods. We chose the (template) star HD 128620 and determined its classic and new $\log R_{\mathrm{HK}}^{\prime}$ values by artificially spinning it up in steps of $10 \mathrm{~km} \mathrm{~s}^{-1}$ up to a maximum $v \sin (i)$ of $190 \mathrm{~km} \mathrm{~s}^{-1}$, thus creating essentially a rapidly rotating inactive star and performing the analysis as described in Sect. 3.1 and 3.2. In order to study the effects of line smearing we also included an artificial emission feature in the spectrum of HD 128620 and carried out the same analysis as before. The resultant $\log R_{\mathrm{HK}}^{\prime}$ values are displayed in Fig. 8 as a function of $v \sin (i)$. The $\log R_{\mathrm{HK}}^{\prime}$ values derived from the MWO method are represented by the bold solid lines while the values derived from the template method are shown as dotted lines. As Fig. 8 demonstrates our new method yields the same $\log R_{\mathrm{HK}}^{\prime}$ values both for the active and inactive star independent of the rotation rate of the object. In contrast, the observed activity for a low activity star derived with the MWO method increases with increasing rotational velocities due to the filling of the line with flux from the line wings, and decreases for a high activity star with increasing rotational velocities. Specifically, the measured $\log R_{\mathrm{HK}}^{\prime}$ for the unbroadened low activity spectrum is -5.15 , which corresponds to a very inactive stars, given the four activity regions defined by Henry et al. (1996). Between 30 and $40 \mathrm{~km} \mathrm{~s}^{-1}$, the increase of the activity due to the contribution of the line wings shifts this object into the regime of the inactive stars at $\log R_{\mathrm{HK}}^{\prime}=-5.1$. At the rotational velocity of the most rapid rotator in our sample, which is about $180 \mathrm{~km} \mathrm{~s}^{-1}$, we obtain a flux increase of around 0.25 dex, which would classify this star an active star with a $\log R_{\mathrm{HK}}^{\prime}$ value of -4.75 . The $\log R_{\mathrm{HK}}^{\prime}$ value of -4.4 of our active star is seen to decrease with increasing rotational velocity due to the fact that the loss of flux from the broadenend emission core more than compensates the filling up of the lines with the flux from the line wings. Only at the very highest rotational velocities above $150 \mathrm{~km} \mathrm{~s}^{-1}$, where the emission core extremely flattened, the decrease of $\log R_{\mathrm{HK}}^{\prime}$ is stopped and the measured activity stays constant. The overall change of $\log R_{\mathrm{HK}}^{\prime}$ from the unbroadened to highly broadened spectra is, compared to the influence on an inactive star, relatively small, with a $\Delta \log R_{\mathrm{HK}}^{\prime} \simeq 0.2$. 


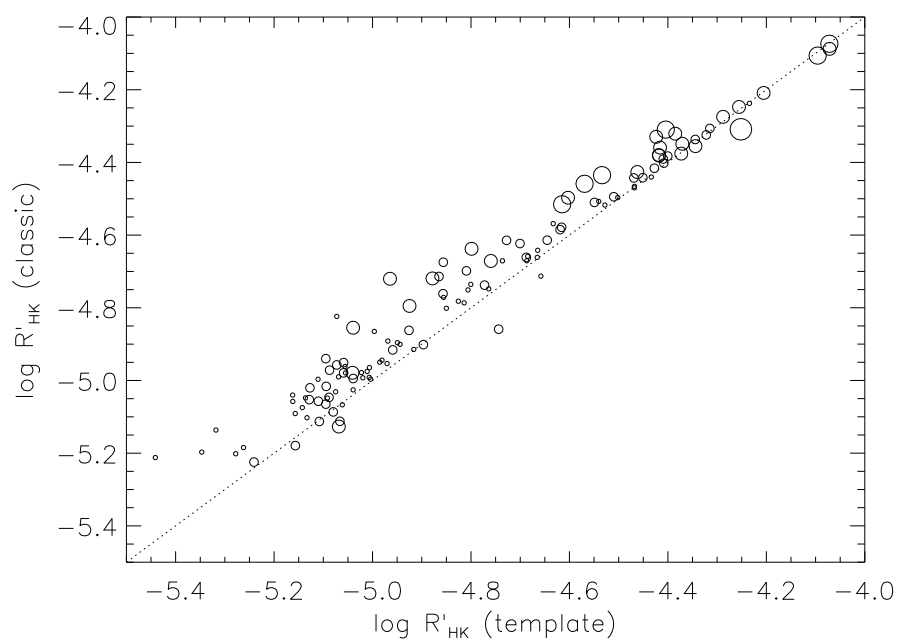

Fig. 9. Classical $R_{\mathrm{HK}}^{\prime}$ values plotted over template $R_{\mathrm{HK}}^{\prime}$ values for mainsequence stars with $B-V \geq 0.44$. The size of the symbols represents the $v \sin (i)$ values of the stars. With increasing $v \sin (i)$, the rotational effect shifts the classical $R_{\mathrm{HK}}^{\prime}$ away from identity to higher values.

The differences between the measurements at $v \sin (i)=$ $0 \mathrm{~km} \mathrm{~s}^{-1}$ are caused by two effects. First, for the inactive star the template method measures no difference between the two (identical) spectra, which results in an $S$-index of 0 and very small $\log R_{\mathrm{HK}}^{\prime}$. Since our lower limit to the $S$-index is actually 0.03 (see Sect. 3.2.1), this translates in a $\log R_{\mathrm{HK}}^{\prime}$ of -5.2 , which is slightly lower than the lower limit of around -5.15 for the classical method. For the active stars, the difference between the template measurement and the classical measurement is caused by uncertainties in the conversion of the $S_{\text {template }}$ into an $S_{\text {template }}^{\prime}$, which is used to calculate the template $\log R_{\mathrm{HK}}^{\prime}$.

We now apply the two methods to real data and compare the difference between the classical $\log R_{\mathrm{HK}}^{\prime}$ values and the $\log R_{\mathrm{HK}}^{\prime}$ values obtained by the template method as shown in Fig. 9. In this plot the size of the symbols represents the rotational velocity of the the star, the step sizes of the symbols are $v \sin (i)$ $<5 \mathrm{~km} \mathrm{~s}^{-1}, 5>v \sin (i)<12 \mathrm{~km} \mathrm{~s}^{-1}, 12>v \sin (i)<30 \mathrm{~km} \mathrm{~s}^{-1}$, $30>v \sin (i)<65 \mathrm{~km} \mathrm{~s}^{-1}$ and $v \sin (i)>65 \mathrm{~km} \mathrm{~s}^{-1}$. The effects of rotational broadening are clearly visible in Fig. 9. For the classical method the measured $\log R_{\mathrm{HK}}^{\prime}$ values strongly depend on the rotational velocity. In the inactive regime the slowly rotating stars are found near the dotted line, which indicates identity. The faster a star rotates, the larger is the distance to the dotted line due to the rotational effect on the inactive stars. In the more active regime the difference becomes smaller even for rapidly rotating stars. This is the same phenomenon as in the measurements for an active star in Fig. 8.

The rotational influence on the $\log R_{\mathrm{HK}}^{\prime}$ values as described above does not cause a problem for most of the solar-like mainsequence stars with $B-V \geq 0.44$, which is the borderline of the previous Ca II H\&K surveys. This is due to the fact that most stars in this color range do not rotate fast enough to suffer a significant change in the measured activity (see Fig. 1). Only the youngest stars, which have not yet lost their angular momentum due to magnetic breaking, rotate fast enough to show a rotational effect in the $\log R_{\mathrm{HK}}^{\prime}$ values. The fastest object in our sample in this color regime is HD 199143, which is an F8V star with $v \sin (i)$ of $125 \mathrm{~km} \mathrm{~s}^{-1}$ and a supposed member of the $\sim 12 \mathrm{Myr}$ old $\beta$ Pic moving group (Kaisler et al. 2004). Since HD 199143 is an highly active star, the effect on the measured activity caused by the rotational velocity is small, because the decrease of flux

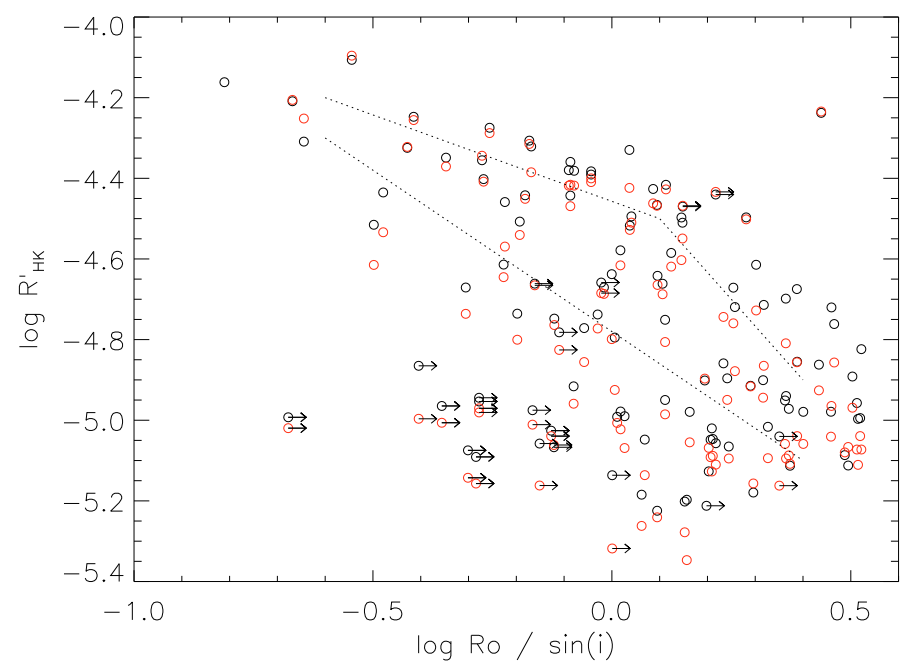

Fig. 10. Classical (black) and template (red) $R_{\mathrm{HK}}^{\prime}$ values for mainsequence stars plotted over the Rossby number. The color range is limited to $B-V>0.44$. Arrows indicate the upper limits for stars with $v \sin (i) \leq 4 \mathrm{~km} \mathrm{~s}^{-1}$. The dotted lines represent the upper and lower activity limit of the sample stars presented by Noyes (1984).

due to the broadening of the emission feature is compensated by the flux of the line wings (see the upper solid line in Fig. 8). However, for the early stars in the range of $B-V \leq 0.4$, the emission is supposed to decrease on the basis of decreasing dynamo efficiency, which leads to a higher impact of the rotational influence. For these stars, the expected effect is described by the lower solid line in Fig. 8.

\subsection{Classical and template log $R_{H K}^{\prime}$ determinations}

Noyes (1984) were the first to empirically show the correlation between Rossby number and activity (as measured through $\log R_{\mathrm{HK}}^{\prime}$ ) and hence provide more or less direct evidence for dynamo related activity in late-type stars. It is clearly desirable to extend these studies to the earliest possible late-type stars. In Fig. 10 we therefore plot the classical and the template $\log R_{\mathrm{HK}}^{\prime}$ values over the Rossby number for those main-sequence stars with $B-V>0.44$. The classical values are represented by black circles and the template value are shown in filled red circles. Evolved objects or stars outside the given color range are left aside for two reasons: First, the convective turnover time is unknown for the evolved stars and becomes very small, according to Eq. (8), for the earliest stars in our sample. The second reason is the fact that the photospheric contribution $R_{\text {phot }}$ is unknown for these stars, too. We also indicated (by the dotted lines in Fig. 10) that area, where the stars in the Noyes (1984) sample are located. In our data we find a number objects at higher Rossby numbers than the values in the Noyes sample. This can be explained by the effects of inclination. Since the periods we used to determine the Rossby number for our sample are calculated from the $v \sin (i)$ values and the inclinations of our sample stars are unknown, the calculated Rossby numbers are by necessity upper limits. In addition to the objects with high Rossby numbers, we see several inactive stars with relatively small log $(R o / v \sin (i))$ values. For these objects, the determined rotational velocity values in the range range of $4 \mathrm{~km} \mathrm{~s}^{-1}$ are in fact upper limits. Therefore, these late $\mathrm{G}$ to $\mathrm{K}$ dwarfs might have lower rotational velocities, which would shift them to higher Rossby numbers. The main point shown by Fig. 10 is the good agreement between the measured $\log R_{\mathrm{HK}}^{\prime}$ values derived with the classical 


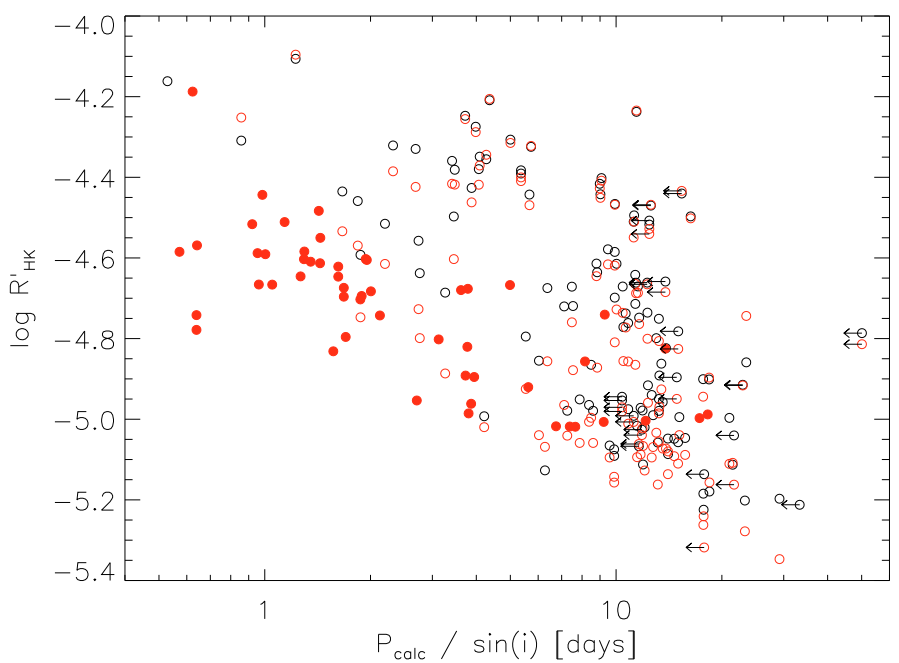

Fig. 11. Classical (black) and template (red) $R_{\mathrm{HK}}^{\prime}$ values for mainsequence stars with plotted over the calculated period. For the classical measurement, the color range is limited to $B-V>0.44$, while the template values are given for all measured main-sequence stars. The filled red circles are the template measurements for stars with $B-V<0.44$. Arrows indicate the upper limits for stars with $v \sin (i) \leq 4 \mathrm{~km} \mathrm{~s}^{-1}$.

and template method for the late $\mathrm{F}$ to $\mathrm{K}$ stars. The differences between the classic and the template values are caused by the rotational effect, as can be seen in Fig. 9. Stars located above the dotted identity line in Fig. 9 are stars with noticeable difference between the classical and the template values in Fig. 10. Given the limitations of our calculated periods we are able to reproduce the rotation-activity relation presented by e.g. Noyes (1984).

Having shown the consistency of our rotation-activity relations for stars with $B-V>0.44$ with previous work we can now extend these relations to stars in the range of $B-V<0.44$ we plot (in Fig. 11) the classical and the template $\log R_{\mathrm{HK}}^{\prime}$ values vs. the calculated period $P_{\text {calc }}$. We now choose the calculated periods as activity parameters since the small convective turn-over times for stars with $B-V<0.44$ lead to very large uncertainties in the inferred Rossby numbers. In Fig. 11 the classical values for stars with $B-V>0.44$ are plotted in black circles, the values for the template method in the same color range in red circles and the template values for the stars with $B-V<0.44$ in filled red circles. As is clear from Fig. 11, the increase in activity towards smaller rotational periods is less steep than in the late F-K stars. For periods longer than 10 days, the early F stars are located in the lower half of the activity distribution. On the other hand, for the rapidly rotating stars with periods of about 1 day, nearly all of these objects are significantly less active than the corresponding later type stars with the same rotation period.

In order to illustrate the color dependence of the derived $\log R_{\mathrm{HK}}^{\prime}$ measurements we show (in Fig. 12) the $\log R_{\mathrm{HK}}^{\prime}$ values (derived from the MWO method) vs. $B-V$ color index. Only stars with $B-V>0.44$ are shown, because for bluer stars the photospheric contribution to the $S$-index is undefined. Using our new template method we can extend this study to bluer stars as shown in Fig. 13, where we plot the $\log R_{\mathrm{HK}}^{\prime}$ values derived from the template method vs. $B-V$ color index. For the stars with $B-V \geq 0.5$ we find a similar distribution of the measured activity as with the Mount Wilson method, however, for the stars with $B-V \leq 0.5$, there is a clear decrease in maximum activity. This decrease in Ca II H\&K activity occurs in the same temperature range as the ratio between $\mathrm{X}$-ray luminosity and bolometric luminosity $L_{X} / L_{\text {bol }}$ (Schmitt et al. 1985) and the fraction of X-ray

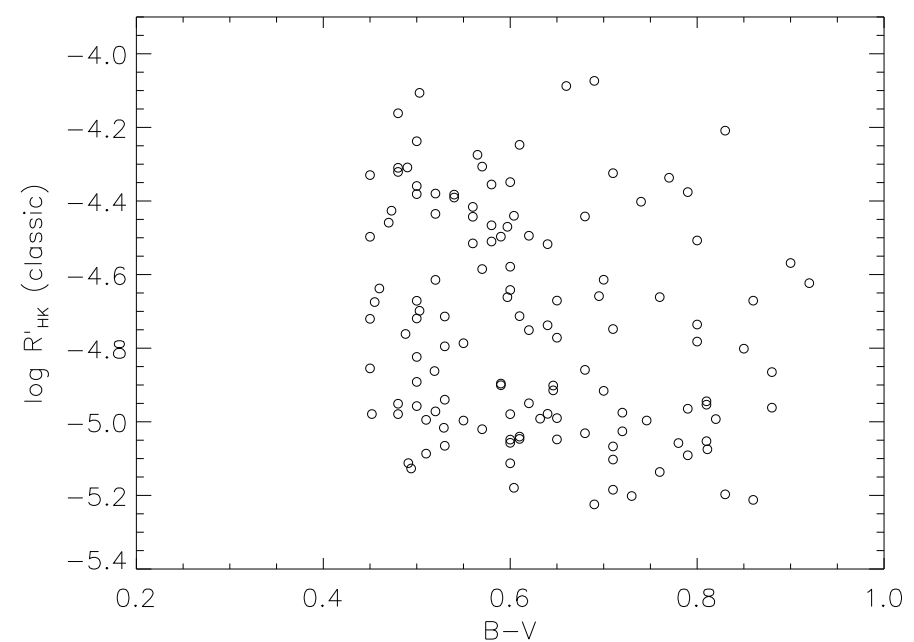

Fig. 12. Classical $R_{\mathrm{HK}}^{\prime}$ values for main-sequence stars plotted over $B-V$ for stars with $B-V \geq 0.44$.

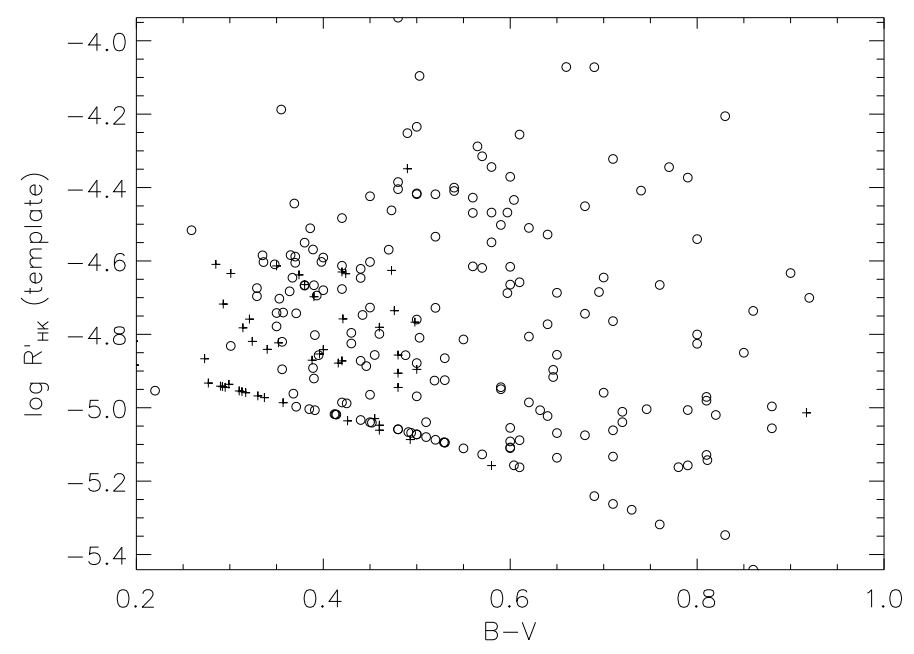

Fig. 13. Template $R_{\mathrm{HK}}^{\prime}$ values plotted over $B-V$. Main-sequence stars are represented by circles, giant stars with "+" symbols. The $B-V$ values range from 0.22 to 0.92 . A clear decrease in Ca II H\&K activity can be seen in the hottest stars of the sample.

detection (Schröder \& Schmitt 2007). The onset of the chromospheric activity is located in the range of $0.1 \leq B-V \leq 0.2$, which corresponds to spectral type A7-8V. We attribute this rise in maximum activity from spectral type A7-8V towards cooler stars to the growing efficiency of the solar-type convective dynamo. To our knowledge this is the first time that this "onset of convection" is directly seen in Ca II H\&K activity, which nicely demonstrates the power of our new template method.

\section{Summary}

In the present paper we measured the chromospheric $\mathrm{Ca}$ II $\mathrm{H} \& \mathrm{~K}$ activity from 481 stars. The observations were carried out with the FEROS and FOCES instruments and include objects from late A- to late K-type stars. From these spectra we extracted the $S$ values according to the Mount Wilson method and calibrated them to the Mount Wilson $S$-index based on a subsample with given $S$-indices from the literature. For main-sequence stars with $B-V>0.44$ we calculated the chromospheric emission ratio $\log R_{\mathrm{HK}}^{\prime}$ and find our measurements to agree with the literature data. Also, the activity distribution in our sample is in agreement 
with previous studies, with a slightly higher fraction of active stars possibly being caused by a bias towards higher rotational velocities in one of the FEROS and the FOCES sample.

Our analysis of the influence of the rotational broadening on the measured $\log R_{\mathrm{HK}}^{\prime}$ shows a minor impact for active stars and a strong impact for rapidly rotating late A to early $\mathrm{F}$ stars, which are supposed to be inactive or little active. This is due to the fact that in the active stars there are two effects caused by rotational broadening, the loss of emission flux in the line core and the filling of the line with flux from the broadened line wings. In active stars both effects approximately cancel out each other, while in the absence of an emission feature in the inactive stars leaves the filling-up of the line as the remaining effect, therefore resulting in high $\log R_{\mathrm{HK}}^{\prime}$ values for these inactive stars.

In order to measure the $\mathrm{Ca}$ II $\mathrm{H} \& \mathrm{~K}$ activity in $\log R_{\mathrm{HK}}^{\prime}$ for stars with $B-V<0.44$, which are often rapidly rotating and relatively inactive, we developed a new method, which is based on the comparison of the target star with a slowly rotating template star. The template spectrum is artificially broadened to the rotational velocity of the star and fitted to the star's spectrum in the line wings. The difference between the template and the star in the line core represents the emission feature and can be converted into $\log R_{\mathrm{HK}}^{\prime}$ values. We selected four stars as template stars, which cover a broad range in $T_{\text {eff }}$ and $\log g$. With these templates, we are able to measure the activity in $\log R_{\mathrm{HK}}^{\prime}$ for 238 sample stars. The fraction of stars in our sample with template measurements can be increased by finding additional template stars. Those stars without a template measurement are stars which differ significantly from our templates in $T_{\text {eff }}$ and/or $\log g$. In two areas of our sample the coverage can be improved: the main-sequence stars in the range of $B-V<0.3$ and $B-V>0.9$. For the first group the main problem is the fact that the number of stars with $v \sin (i)<10 \mathrm{~km} \mathrm{~s}^{-1}$ is very small. Those objects are most likely observed at high inclination angles, which limits the number of template candidates. For the second group the limiting factor is the fact that the template star must be inactive. Considering the fact that the Mount Wilson method does not suffer from the problems, which are present in the early solar-like stars when applied to the late solar-like stars, the main field of application of our template method are clearly the rapidly rotating late A- to F-type stars. For these objects our new method produces results contributing consistent measurements to existing chromospheric emission measurements. Specifically, our measurements of the chromospheric emission ratio showed, for the first time to our knowledge, the onset of the solar-type dynamo at late A- and early F-type stars in $\log R_{\mathrm{HK}}^{\prime}$ values.

\section{References}

Allen, C. W. 1976, Astrophysical Quantities (London: Athlone, 3rd Ed.) Baliunas, S. L., Donahue, R. A., Soon, W. H., et al. 1995, ApJ, 438, 269

Baliunas, S. L., Donahue, R. A., Soon, W., \& Henry, G. W. 1998, in Cool Stars, Stellar Systems, and the Sun, ed. R. A. Donahue, \& J. A. Bookbinder, ASP Conf. Ser., 154, 153

Cincunegui, C., Díaz, R. F., \& Mauas, P. J. D. 2007, A\&A, 469, 309

Duncan, D. K., Vaughan, A. H., Wilson, O. C., et al. 1991, ApJS, 76, 383

Glebocki, R., \& Stawikowski, A. 2000, Acta Astron., 50, 509

Gray, R. O., Corbally, C. J., Garrison, R. F., McFadden, M. T., \& Robinson, P. E. 2003, AJ, 126, 2048

Gray, R. O., Corbally, C. J., Garrison, R. F., et al. 2006, AJ, 132, 161

Hall, J. C., Lockwood, G. W., \& Skiff, B. A. 2007, AJ, 133, 862

Hauschildt, P. H. \& Baron, E. 1999, J. Comp. Appl. Math., 109, 41

Henry, T. J., Soderblom, D. R., Donahue, R. A., \& Baliunas, S. L. 1996, AJ, 111, 439

Kaisler, D., Zuckerman, B., Song, I., et al. 2004, A\&A, 414, 175

Kaufer, A., Stahl, O., Tubbesing, S., et al. 1999, The Messenger, 95, 8

Middelkoop, F. 1982, A\&A, 107, 31

Moon, T. T., \& Dworetsky, M. M. 1985, MNRAS, 217, 305

Napiwotzki, R., Schönberner, D., \& Wenske, V. 1992, in The Atmospheres of Early-Type Stars, ed. U. Heber, \& C. S. Jeffery, Lect. Not. Phys. (Berlin: Springer Verlag), 401, 18

Nordström, B., Mayor, M., Andersen, J., et al. 2004, A\&A, 418, 989

Noyes, R. W. 1984, Adv. Space Res., 4, 151

Reiners, A., \& Schmitt, J. H. M. M. 2002, A\&A, 384, 155

Reiners, A., \& Schmitt, J. H. M. M. 2003a, A\&A, 412, 813

Reiners, A., \& Schmitt, J. H. M. M. 2003b, A\&A, 398, 647

Relyea, L. J., \& Kurucz, R. L. 1978, ApJS, 37, 45

Rutten, R. G. M. 1984, A\&A, 130, 353

Schmidt, E. G., \& Taylor, D. J. 1979, AJ, 84, 1193

Schmitt, J. H. M. M., Golub, L., Harnden, Jr., F. R., et al. 1985, ApJ, 290, 307

Schröder, C., \& Schmitt, J. H. M. M. 2007, A\&A, 475, 677

Vaughan, A. H., Preston, G. W., \& Wilson, O. C. 1978, PASP, 90, 267

Wilson, O. C. 1978, ApJ, 226, 379

Wright, J. T., Marcy, G. W., Butler, R. P., \& Vogt, S. S. 2004, ApJS, 152, 261 